\title{
Apoptosis-inducing factor and calpain upregulation in glutamate-induced injury of rat spiral ganglion neurons
}

\author{
ZHONG-JIA DING $^{1}$, XIN CHEN $^{1}$, XIAO-XU TANG ${ }^{2}$, XI WANG $^{1}$, YONG-LI SONG ${ }^{1}$, XIAO-DONG CHEN ${ }^{1}$, \\ JIAN WANG $^{1}$, REN-FENG WANG ${ }^{1}$, WEN-JUAN MI ${ }^{1}$, FU-QUAN CHEN ${ }^{1}$ and JIAN-HUA QIU ${ }^{1}$ \\ ${ }^{1}$ Department of Otolaryngology-Head and Neck Surgery, Xijing Hospital, Fourth Military Medical University, \\ Xi'an, Shaanxi 710032; ${ }^{2}$ Outpatient Department, Logistics Academy, Beijing 100858, P.R. China
}

Received May 30, 2014; Accepted February 27, 2015

DOI: $10.3892 / \mathrm{mmr} .2015 .3626$

\begin{abstract}
Spiral ganglion neuron (SGN) damage and apoptosis can lead to noise-induced hearing loss, age-associated hearing loss and, in certain cases, auditory neuropathy. The apoptosis-inducing factor (AIF)-associated pathway may be important in this process. The present study aimed to investigate the expression levels of AIF and calpain in damaged SGNs. Glutamate (Glu) perfusion and cell culture in different concentrations of Glu were performed to damage the SGNs of Sprague-Dawley (SD) rats, with saline water used as a control Different concentrations $(5,10,20$ and $40 \mathrm{mM})$ of Glu were injected into the cochlear tympanic canal of $18 \mathrm{SD}$ rats, and 10, 20 and $40 \mathrm{mM}$ Glu were added to SGN cultures. Auditory brainstem responses (ABR) were measured prior to and 2 days following the injection of Glu. Immunofluorescent staining was used to detect the SGN damage and the expression levels of AIF and calpain in vivo and in in vitro. Transmission electron microscopy (TEM) was used to measure cell apoptosis and reverse transcription-quantitative polymerase chain reaction was used to analyse the gene expression levels of AIF and calpain in the damaged SGNs. The TEM identified mitochondrial vacuolisation, swelling of the SGN and heterochromatin formation. Injection of Glu reduced the number of SGNs and induced apoptosis. AIF was observed to translocate into the nuclei of the SGNs in the 20 and $40 \mathrm{mM}$ Glu groups, and the expression levels of AIF and calpain were markedly upregulated in the modiolus of the Glu-damaged SGNs. The upregulation of AIF and calpain may be important in the process of SGN damage and apoptosis.
\end{abstract}

Correspondence to: Mr. Jian-Hua Qiu or Mr. Fu-Quan Chen, Department of Otolaryngology-Head and Neck Surgery, Xijing Hospital, Fourth Military Medical University, 15 Changle Western Road, Xi'an, Shaanxi 710032, P.R. China

E-mail: qiujh@fmmu.edu.cn

E-mail: chenfq@yahoo.com

Key words: glutamate, perfusion, culture, cochlea, spiral ganglion neurons, apoptosis-inducing factor, calpain

\section{Introduction}

Apoptosis-inducing factor (AIF) is a flavoprotein, which usually performs a redox reaction in the electron transport chain (1) and induces apoptosis under conditions of injury. AIF is cleaved by calpain is and released from the mitochondria into the cytoplasm, where it relocates to the nucleus, cleaves DNA into fragments and induces apoptosis $(2,3)$. AIF is commonly expressed in cells, however, apoptosis is not induced in all types of cell (4), only in neuronal cells and certain tumour cells. Calpains belong to a family of calcium-dependent, non-lysosomal cysteine proteases, which are expressed ubiquitously in all cells (5). Regulated by the amount of $\mathrm{Ca}^{2+}$, calpain has been previously reported to promote apoptosis $(5,6)$. Apoptosis is central in neural injury disease, including spiral ganglion neuron (SGN) injury (7). SGNs, as the first afferent neuron in the auditory pathway, have become a focus of investigation. SGNs are susceptible to damage and the induction of apoptosis by noise, ischemia and hypoxia, which can lead to noise-induced hearing loss, age-associated hearing loss and even auditory neuropathy $(8,9)$.

In the cochlea, SGN damage and apoptosis are regulated by a complex signaling pathway. Fu et al perfused ouabain into the cochlea to induce SGN apoptosis via the expression of caspase (9). Schmutzhard demonstrated that severe sepsis-induced hearing loss can be attributed to apoptosis of the supporting cells, mediated by an upregulation of caspase 3 (10). However, inhibition of caspases results in incomplete or limited protection, and AIF is concentrated in cochlea sensory cells. A previous study observed that $122 \mathrm{~dB}$ white noise induces the relocation of AIF into the nuclei (11).

Therefore, in the present study, Glu was directly perfused into the cochlear tympanic canal (12) and SGNs were cultured in vitro in order to examine the expression levels of AIF and calpain in Glu-damaged SGNs.

\section{Materials and methods}

Animals and ethical statement. A total of 18 female Sprague-Dawley rats, aged 4-6 weeks and weighing between 150 and $180 \mathrm{~g}$, were used for liquid perfusion, and postnatal 0-3-day old rats were used for the in vitro culture of SGNs. 
The rats were maintained in clean conditions, with ad libitum access to food and water at $37^{\circ} \mathrm{C}$ and $40 \%$ humidity. All animals were provided by and cared for by the Institutional Animal Care and Use Committee of the Fourth Military Medical University (Xi'an, China). The present study was approved by the Ethics Committee of Xijing Hospital (Xi'an, China).

Liquid perfusion. The rats for the liquid perfusion analysis were administered $2 \%$ sodium pentobarbital $(0.25 \mathrm{ml} / 100 \mathrm{~g}$; Sigma-Aldrich, St. Louis, MO, USA) to induce anaesthesia, and placed on a board at $37^{\circ} \mathrm{C}$ to maintain their temperature. The skin and fascia surrounding the ear were dissected, and the muscles were separated to expose the otocyst. A power drill (XiChang Inc., Xi'an, China) was used to drill a hole in the otocyst, revealing the cochlea and the artery at the top of the hole. A hole ( $0.2 \mathrm{~mm}$ in diameter) was then opened on the wall of the scala tympani using a hand drill (XiChang Inc.). A plastic tube connected to a Hamilton syringe pump (XiChang Inc.) was used to inject different concentrations of Glu (Hexin Chemical Industry, Shenzhen, China) for $30 \mathrm{~min}$. Subsequently, the wound was closed. All the animals were divided into six groups, which received either injected saline water (control), 5, 10, 20 or $40 \mathrm{mM}$ glutamate, or no treatment. In the control animals, the normal saline was perfused into the perilymph via the same procedure and in all rats, with the exception of the untreated rats, surgery was performed on both ears.

Auditory brainstem response (ABR). The auditory thresholds were determined by measuring the ABR. A TDT III System Auditory Evoked Potential Workstation, controlled using SigGenRZ and BioSigRZ software (Tucker-Davis Technologies, Fort Lauderdale, FL, USA) was used to collect data. The ABRs were elicited using tone bursts $(4,8,16$, 24 and $32 \mathrm{kHz}$; $0.5 \mathrm{~ms}$ rise/fall time; no plateau; alternating phase). The stimulus was provided through an RZ6 D/A converter (Tucker-Davis Technologies) and was presented using a high-frequency speaker (MF1 Multi-Field Magnetic Speakers; Tucker-Davis Technologies) placed $\sim 2 \mathrm{~cm}$ in front of the ear being assessed. The stimulus was reduced in $5 \mathrm{~dB}$ steps until the response ceased. The differential potential was sampled over $10 \mathrm{~ms}$, filtered (low-pass, $4 \mathrm{kHz}$; high-pass, $100 \mathrm{~Hz}$ ) and averaged (512 sweeps of alternating stimulus polarity) in order to obtain the mean traces at each intensity. The rats were assessed 2 days following the perfusion, and the resulting data were analysed using SPSS version 17.0 software (SPSS, Inc., Chicago, IL, USA).

SGNs culture. Dissociated spiral ganglion cultures were prepared, as described previously, from postnatal 0-3 day rats (13). In brief, the SGNs $\left(\sim 1 \times 10^{4}\right)$ were incubated in SGN culture medium: Dulbecco's modified Eagle's medium with B27 (2 ml/ml; Sigma-Aldrich), brain-derived neurotrophic factor $(10 \mu \mathrm{g} / \mathrm{ml}$; Sigma-Aldrich), penicillin $(100,000 \mathrm{U} / \mathrm{l}$; $1 \%$; Sigma-Aldrich). Subsets of the cultures were maintained at $37^{\circ} \mathrm{C}$ in a humidified incubator (Heraeus $\mathrm{CO}_{2}$; Bole Company, Beijing, China) with $5 \% \mathrm{CO}_{2}$, which was divided into four groups, in which either 10, 20 or $40 \mathrm{mM}$ Glu was added to cells for $48 \mathrm{~h}$ at $37^{\circ} \mathrm{C}$.
Immunofluorescent staining. The slides were fixed by perfusing with $4 \%$ paraformaldehyde (XiChang Inc.) for 1 day, following which, the slides were immersed in 0.1 M phophate-buffered saline (PBS; Huamei Biotech Co., Ltd.) for $20 \mathrm{~min}$ and Triton X-100 (0.3\%; Sigma-Aldrich) for $15 \mathrm{~min}$. The slides were washed with $0.1 \mathrm{M}$ PBS for $5 \mathrm{~min}$ each time and were then incubated at $37^{\circ} \mathrm{C}$ in a $5 \%$ goat-serum blocking solution (Huamei Biotech Co., Ltd., Wuhan, China) for $30 \mathrm{~min}$. The slides were incubated with primary antibodies against AIF (polyclonal goat; 1:100; Santa Cruz Biotechnology, Inc., Dallas, TX, USA) and $\beta$-tubulin (polyclonal rabbit; 1:200; Abcam, Cambridge, MA, USA) at $4^{\circ} \mathrm{C}$ in a refrigerator for 3 days. The slides were then washed with $0.1 \mathrm{M}$ PBS and were incubated with Alexa Fluor 488 (polyclonal donkey anti-goat; 1:200; Invitrogen Life Technologies, Carlsbad, CA, USA) and Cy3 (polyclonal goat anti-rabbit; 1:100; Abcam) at $4^{\circ} \mathrm{C}$ for 1 day. Subsequent to the addition of $0.1 \%$ DAPI (Sigma-Aldrich) to stain the nuclei, glycerol (Huamei Biotech Co., Ltd.) was used to seal the coverslips. The sections were observed using a laser scanning confocal microscope (FV1000; Olympus Corporation, Tokyo, Japan).

Transmission electron microscopy (TEM). The treated rats were fixed using a $2.5 \%$ glutaraldehyde (Huamei Biotech Co., Ltd.) solution. The rats were sacrificed by decapitation, and the cochlea was removed from the head and immersed in the same fixative at $4^{\circ} \mathrm{C}$ overnight. The specimens were then rinsed with 0.1 M PBS and immersed in a 10\% EDTA (Sigma-Aldrich) solution for 7 days for decalcification. Subsequently, the cochlea was placed in a $1 \%$ osmic acid solution (Abcam) for $2 \mathrm{~h}$ at $4^{\circ} \mathrm{C}$. The entire cochlea was removed from the bony shell, embedded and cut into ultrathin transverse sections (70-100 $\mathrm{nm}$ ) using an ultramicrotome (Reichert UltracutE, Leica, USA). Counterstaining was then performed using uranyl acetate (Huamei Biotech Co., Ltd.) for $50 \mathrm{~min}$ and lead citrate (Huamei Biotech Co., Ltd.) for 10 min. Finally, the specimens were observed using a TEM (JEM-1230; Olympus Corporation).

Reverse transcription-quantitative polymerase chain reaction $(R T-q P C R)$. The RNA from the treated cochleae were used for RT-qPCR, according to the QuantiTect Reverse Transcription (Qiagen, Valencia, CA, USA) instructions. RT-qPCR analysis was performed using a SYBR ${ }^{\circledR}$ Green Master Mix kit (Applied Biosystems Life Technologies, Foster City, CA, USA) and RNase-free 96-well PCR plates. The qPCR was performed on a CFX96 Touch $^{\mathrm{TM}}$ Real-Time PCR Detection system (Bio-Rad Laboratories, Inc., Hercules, CA, USA). The following forward and reverse primers (Genecopoeia, Rockford, MD, USA) were used for the specific RNAs in RT-qPCR: R-AIF, forward 5'-TAGAACTCCAGATGGCAAGACA-3'; R-AIF, reverse 5'-AAGCCCACAATAAGGACTAACAC-3'; R-caspase 3, forward 5'-GAATGACTGGGAGTGGGGTAG-3'; R-caspase 3, reverse 5'-GACCTGGAACATCGGATTTGA-3'; R-calpain, forward 5'-CAAAGTGGACCCCTATGAACG-3'; R-calpain, reverse 5'-TAAGGGCGTCAGGTGTAAGGT-3'. The threshold cycles $(\mathrm{Ct})$ value of the genes under examination in each sample were normalised using the value of the endogenous control gene, $18 \mathrm{~S}$. The relative fold changes in gene expression levels were obtained by comparing the $2^{-\Delta \Delta \mathrm{Ct}}$ data of the different groups. 


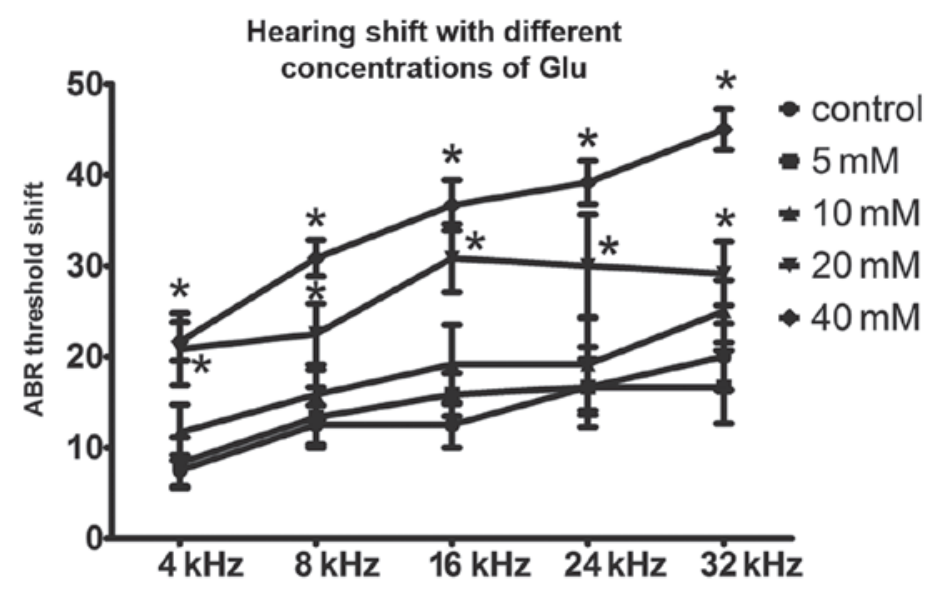

Figure 1. Hearing shift data subsequent to the perfusion with different concentrations of Glu. The average ABR thresholds were increased at $4,8,16,24$ and $32 \mathrm{kHz}$. No significant differences were observed between the control group and the 5 and 10 mM groups, however, significant increases were observed in the 20 and $40 \mathrm{mM}$ groups. Higher concentrations of Glu perfusion were associated with higher shifts in frequencies. ( $\mathrm{P}<0.05$, vs. $5 \mathrm{mM}, 10 \mathrm{mM}$ and control groups). Data are expressed as the mean \pm standard deviation. Glu, glutamate; ABR, auditory brainstem responses.

Data analysis. Wherever possible, the data are expressed as the mean \pm standard deviation. Statistical significance was determined using analysis of variance followed by a multiple comparison Dunnett's test (using SPSS software). $\mathrm{P}<0.05$ was considered to indicate a statistically significant difference.

\section{Results}

ABR thresholds increase following Glu perfusion. Subsequent to Glu perfusion, the ABR threshold was markedly increased. No significant difference was observed among the values for the control, 5 or $10 \mathrm{mM}$ Glu groups ( $\mathrm{P}>0.05)$, however, a significant increase was observed in the 20 and $40 \mathrm{mM}$ Glu groups $(\mathrm{P}<0.05)$. In the control and $5 \mathrm{mM}$ groups, there was a $7-8 \mathrm{~dB}$ shift at $4 \mathrm{kHz}$, in the $10 \mathrm{mM}$ group, the shift reached $11 \mathrm{~dB}$, and in the 20 and $40 \mathrm{mM}$ groups, the shift reached $20 \mathrm{~dB}$. At 8 and $16 \mathrm{kHz}$, the shift in the control and $5 \mathrm{mM}$ groups was $12-15 \mathrm{~dB}$, however, there was a shift of 20-30 dB in the 10, 20 and $40 \mathrm{mM}$ groups. At 24 and $32 \mathrm{kHz}$, the shift was $<20 \mathrm{~dB}$ in the control, 5 and $10 \mathrm{mM}$ groups, but was $>20 \mathrm{~dB}$ in the other groups. The ABR shifts increased with the frequency of the toneburst (Fig. 1).

Glutamate toxicity reduces the number of cochlear SGNs and alters their morphology. A total of three successive sections of the cochlea middle axis were selected for tubulin staining. Images were captured using a laser scanning confocal microscope and the mean number of basal SGNs present in each group were counted by two or more individuals. The Glu perfusion groups were compared with the control, providing the rates of SGN loss. The results demonstrated that the mean number of SGNs was reduced by a magnitude of between two and three in the 5 and $10 \mathrm{mM}$ Glu groups, compared with the control group, and by 8-9-fold in the 20 and $40 \mathrm{mM}$ Glu groups (data not shown). The statistical analyses revealed that the numbers of SGNs in the latter groups were significantly lower compared with those in the that of the 5 and $10 \mathrm{mM}$ Glu groups (Fig. 2). TEM also revealed that treatment with Glu at $\geq 10 \mathrm{mM}$ generated a high number of cytoplasmic vacuoles and a significant quantity of heterochromatin around the nuclei in the SGNs, in addition to swelling of the mitochondria and endoplasmic reticulum (Fig. 3). It was, therefore, concluded that excessive Glu damaged the SGNs in the cochlea, in a dose-dependent manner.

Expression levels of caspase 3 are unchanged, whereas those of AIF and calpain are altered in Glu toxicity-induced SGNs. The perfused rats were decapitated on a clean bench, Rosenthal's canal and the middle axis were separated and the basilar membrane and spiral ligament were removed. RT-qPCR was performed to detect the expression levels of AIF, calpain and caspase 3. Increased expression levels of AIF were observed in the Glu intervention groups, whereas the expression levels of caspase 3 were not significantly different. Thus, SGN damage did not upregulate the expression of caspase 3. Changes in the expression levels of calpain were also observed in all the treatment groups. In the 5 and $10 \mathrm{mM}$ Glu groups, the expression levels of calpain were significantly higher compared with those of the $20,40 \mathrm{mM}$ and control groups $(\mathrm{P}<0.05$; Fig. 4). In conclusion, AIF, rather than caspase 3, initiated the Glu toxicity-induced SGN apoptosis. Calpain was also involved in the damage of the SGNs.

AIF is translocated into the nuclei following exposure to Glu at concentrations $\geq 20 \mathrm{mM}$. To assess the localisation and expression levels of AIF, the subcellular location of AIF was examined in SGNs following Glu perfusion. The results demonstrated that, in the groups treated with $\geq 20 \mathrm{mM}$ Glu, the red fluorescence of AIF overlapped with the blue nuclear staining in the laser scanning confocal microscopy; however, concentric circles of red labelling surrounded the blue labelling were observed in the control group. The staining patterns of the perfused groups were irregular and included incomplete nuclear staining and polygonal cytoplasmic labelling. These altered patterns of expression suggested that the AIF was initally translocated into the nuclei of the SGNs (Fig. 5).

AIF is translocated into the nucleus and the expression levels of AIF and calpain, but not caspase 3 are upregulated in Glu-treated SGNs in vitro. To confirm the mRNA expression 

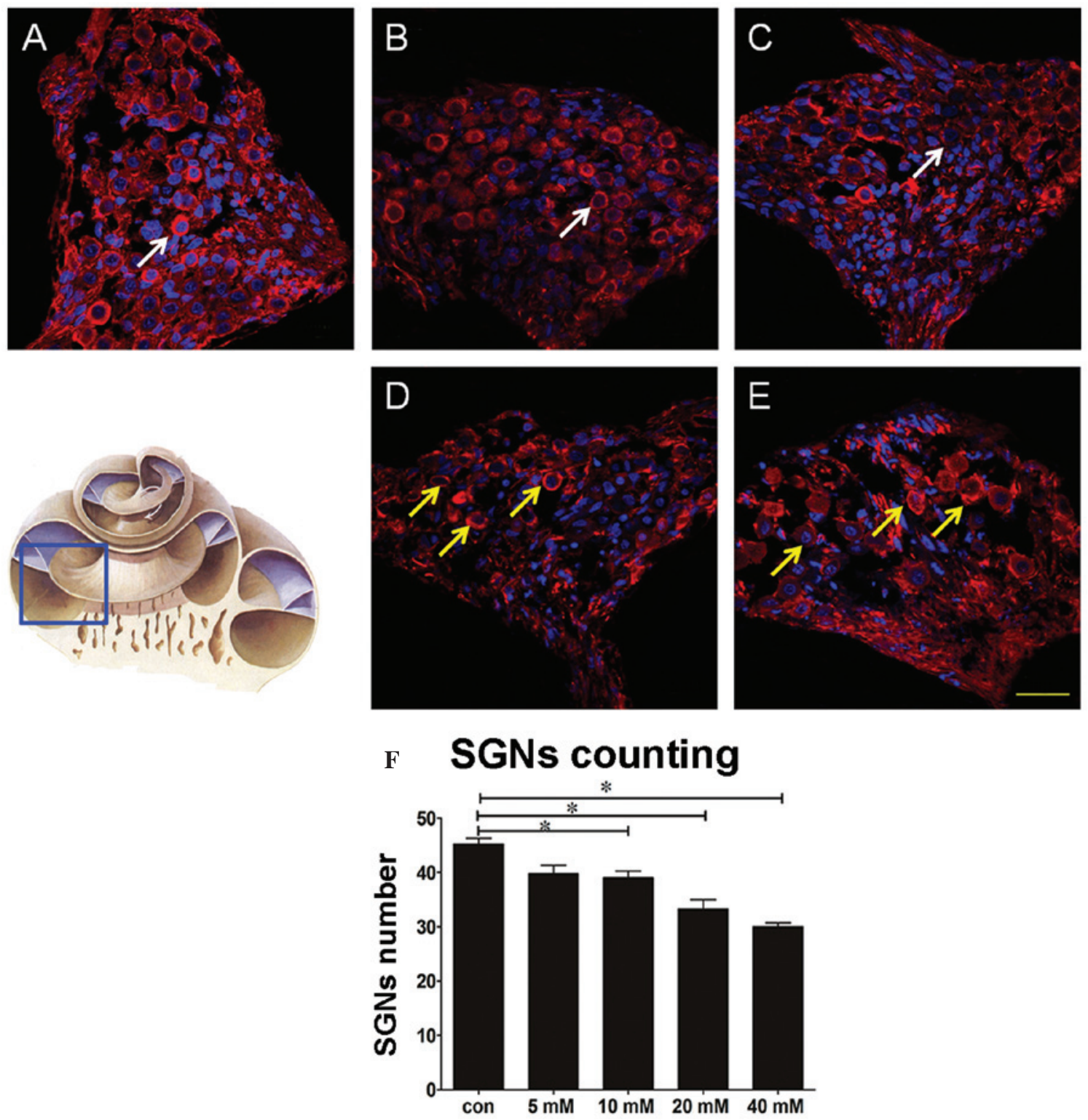

Figure 2. Morphology of the SGNs and the proportion of remaining SGNs compared with the control group. Tubulin (red) indicates the morphology of the SGNs, and DAPI (blue) indicates the nuclei. Immunofluorescence images in the (A) control group and at Glu perfusion concentrations of (B) 5, (C) 10, (D) 20 and (E) $40 \mathrm{mM}$. (F) Histogram depicting the proportion of SGNs lost in each image. White arrows indicate normal SGN; yellow arrows indicate abnormal SGN or irregular morphology. Scale bar, $50 \mu \mathrm{m}$. Data are expressed as the mean \pm standard deviation. SGNs, spiral ganglion neurons; Glu, glutamate; con, control.

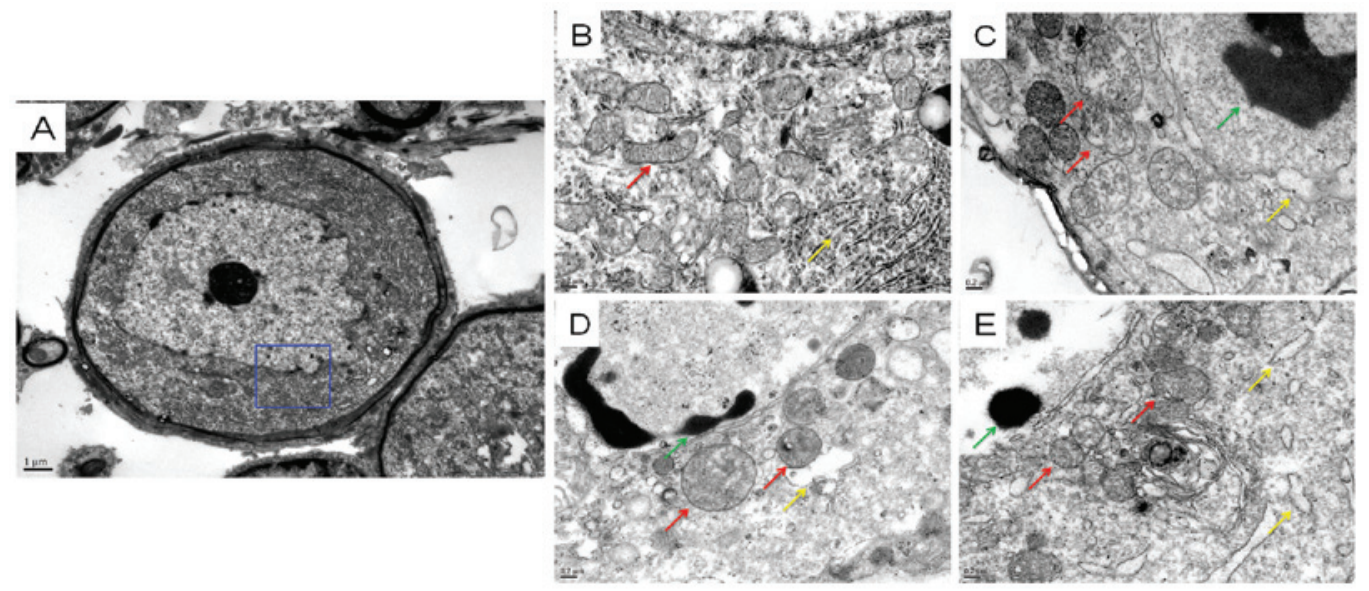

Figure 3. Morphology of SGNs in the Glu perfusion groups via transmission electron microscopy. Images of the SGNs in the (A) control cells and at Glu perfusion concentrations of (B) 5, (C) 10, (D) 20 and (E) $40 \mathrm{mM}$ (magnification, x40,000). Yellow arrows indicate the endoplasmic reticulum, red arrows indicate the mitochondria and green arrows indicate the heterochromatin gathered around the nuclear membrane. Swelling and vacuoles were observed in (C), (D) and (E). Scale bar, $50 \mu \mathrm{m}$. SGNs, spiral ganglion neurons; Glu, glutamate. 
A

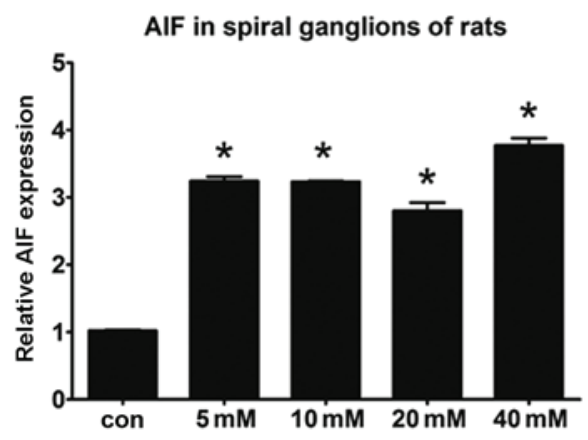

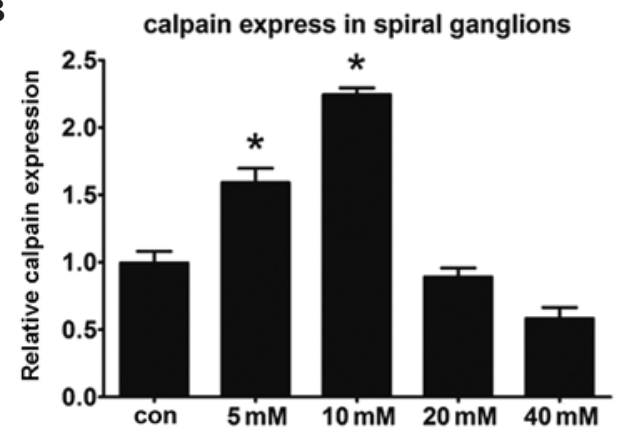

C

caspase 3 expression in spiral ganglions

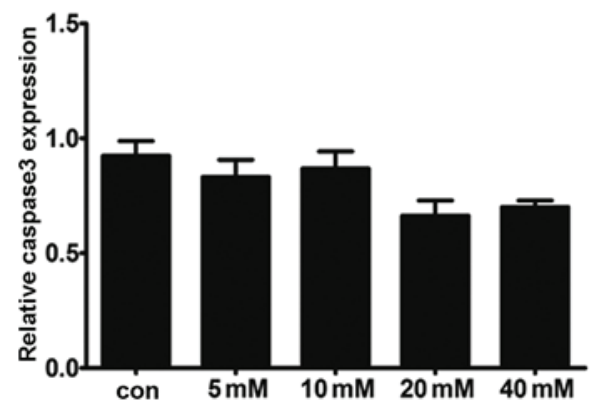

Figure 4. Reverse transcription-quantitative polymerase chain reaction results for AIF, caspase 3 and calpain following Glu perfusion. The expression levels of (A) AIF, (B) calpain and (C) caspase 3 in all the treatment groups are shown. The AIF histogram reveals upregulation of AIF in all the Glu perfusion groups, compared with the control group. The calpain histogram reveals significant upregulation in the $5 \mathrm{mM}$ and $10 \mathrm{mM}$ groups, compared with the other groups. ("P<0.05). No significant differences were observed between the expression levels of caspase 3 in the Glu perfusion groups and in the control group. The $\Delta \Delta \mathrm{Ct}$ method was used to measure the mRNA transcription levels in the SGNs. Data are expressed as the mean \pm standard deviation. AIF, apoptosis-inducing factor; Glu, glutamate; con, control; SGNs, spiral ganglion neurons.
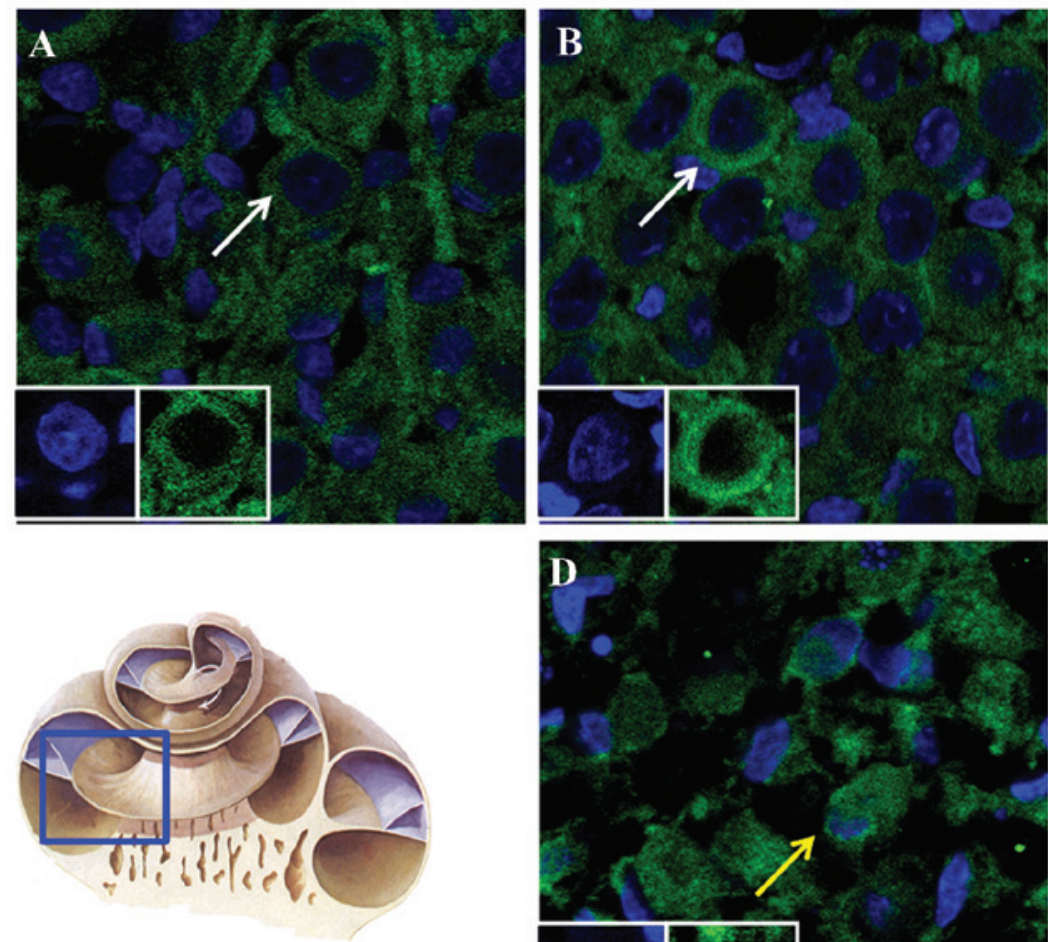
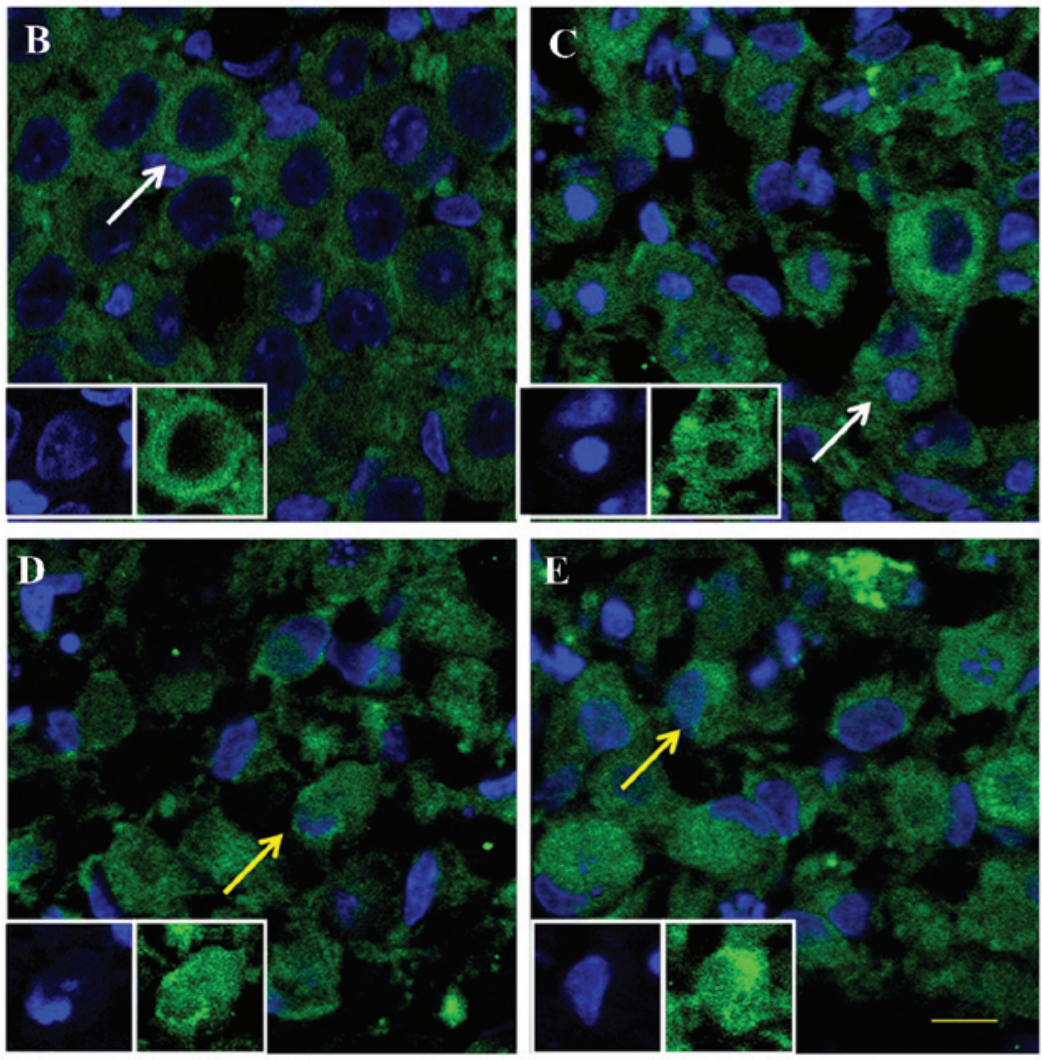

Figure 5. Immunofluorescence results of the distribution of AIF in SGNs perfused with Glu. Immunofluorescence images at in the (A) control, and at Glu perfusion concentrations of (B) 5, (C) 10, (D) 20 and (E) $40 \mathrm{mM}$. Scale bar, $50 \mu \mathrm{m}$. AIF (green) is located in the cytoplasm in the normal group, whereas the nuclei (blue) are visible in the central regions of the SGNs. In the $10 \mathrm{mM}, 20 \mathrm{mM}$ and $40 \mathrm{mM}$ groups, the green and blue signals overlap. White arrows indicate cells, in which AIF was distributed in the cytoplasm, and yellow arrows indicate cells, in which AIF overlapped with the nuclei. AIF, apoptosis-inducing factor; SGNs, spiral ganglion neurons; Glu, glutamate. 
A

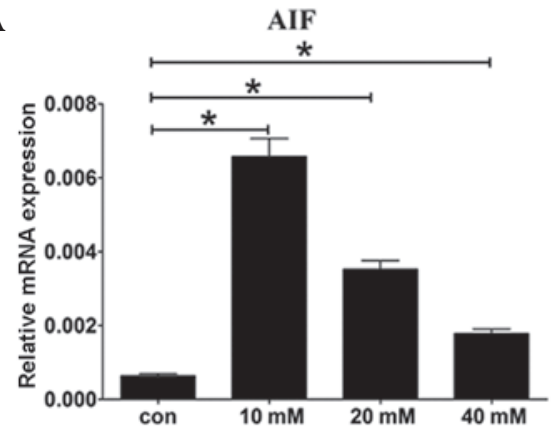

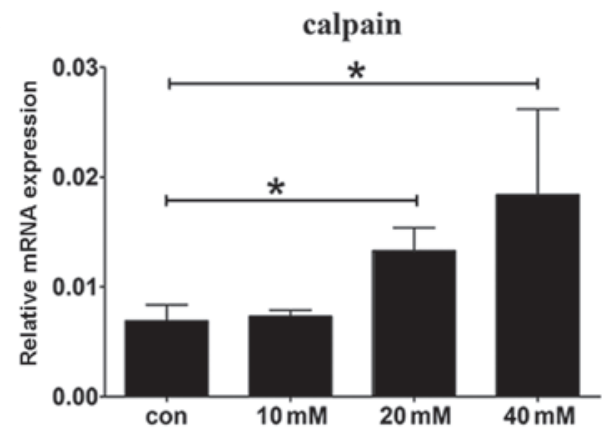

caspase 3

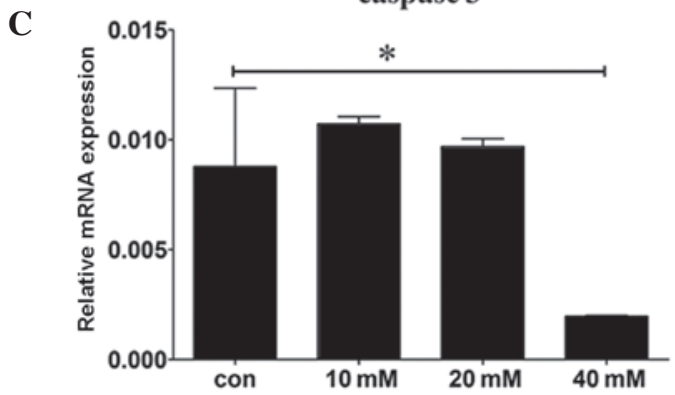

Figure 6. Reverse transcription-quantitative polymerase chain reaction results of AIF, caspase 3 and calpain in cultured SGNs. (A) mRNA expression of AIF was increased following Glu intervention, with significant increases in expression observed in the 10,20 and $40 \mathrm{mM}$ groups (P<0.05 vs. con). (B) mRNA expression of calpain was also upregulated, with significant increases in the 20 and $40 \mathrm{mM}$ groups ( $\mathrm{P}<0.05 \mathrm{vs}$. con). (C) Expression of caspase 3 was unhanged following intervention, whereas the expression in the $40 \mathrm{mM}$ group was significantly reduced ( $\mathrm{P}<0.05 \mathrm{vs}$. con). Data are expressed as the mean \pm standard deviation. AIF, apoptosis-inducing factor; SGNs, spiral ganglion neurons.
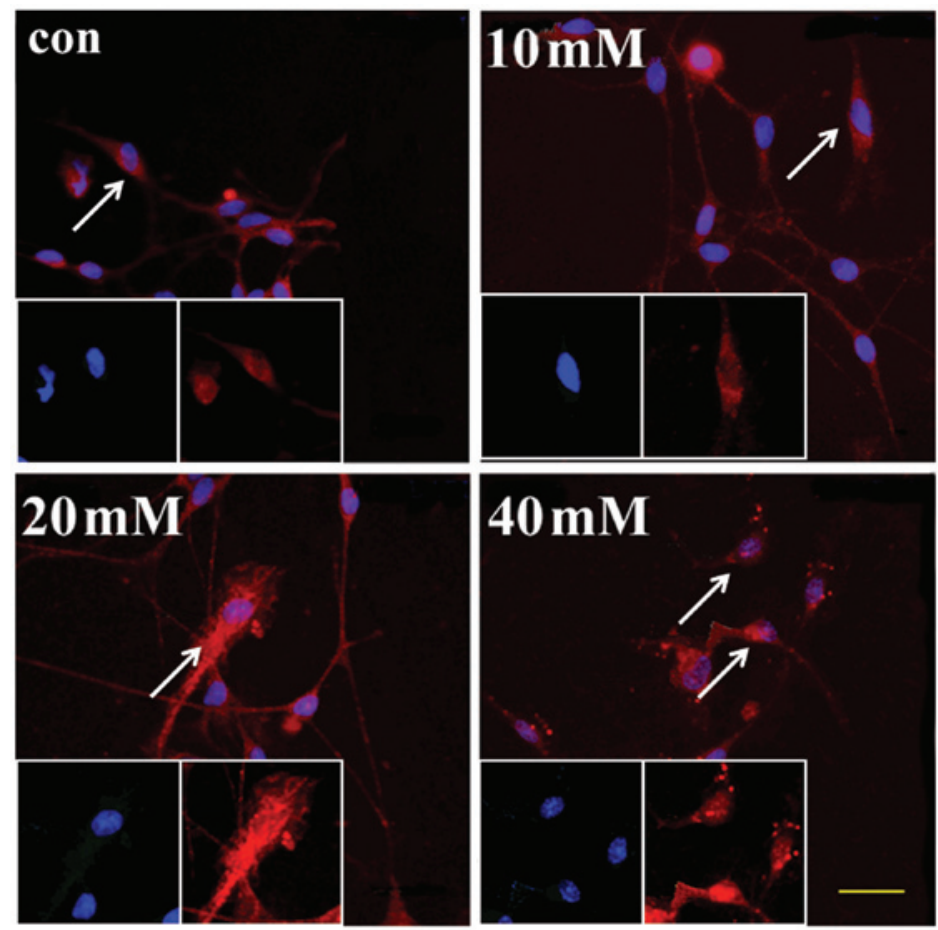

Figure 7. Immunofluorescence results of the distribution of AIF in cultured SGNs. AIF was stained red and the nuclei were stained blue. In the control and $10 \mathrm{mM}$ groups, AIF was distributed in the cytoplasm without overlap of the blue nuclei. However, AIF overlapped with nuclei in the $20 \mathrm{mM}$ group (white arrow), which demonstrated that AIF had translocated into the nuclei. In the $40 \mathrm{mM}$ group, the levels of AIF in the nuclei were increased. AIF, apoptosis-inducing factor; SGNs, spiral ganglion neurons.

levels of AIF and calpain, the SGNs were incubated in medium, containing different concentrations of Glu. The mRNA expression levels of AIF and calpain increased following the addition of Glu for $48 \mathrm{~h}$, and the expression levels of AIF were significantly higher, compared with the control, in the 10,20 and $40 \mathrm{mM}$ groups $(\mathrm{P}<0.05)$. The expression levels of calpain were also higher in the 20 and $40 \mathrm{mM}$ groups compared with the control $(\mathrm{P}<0.05)$. However, the expression levels of 
caspase 3 were not significantly different in the four groups $(\mathrm{P}>0.05)$. These results demonstrated that AIF and calpain were upregulated in Glu-treated SGNs in vitro, however, the levels of caspase 3 were unchanged (Fig. 6).

To observe the distribution of AIF, an immunostaining assay was performed. In the control group, the red-stained AIF was distributed in the cytoplasm only. However, in the $20 \mathrm{mM}$ group, AIF had translocated into the nuclei of certain bipolar neurons, which was observed in greater number of cells in the $40 \mathrm{mM}$ group (Fig. 7). In conclusion, the addition of a high concentration of Glu induced the translocation of AIF into the nuclei, and the upregulation of AIF and calpain in the cultured SGNs.

\section{Discussion}

Spiral ganglia, as the first afferent nerves, transmit acoustic information to the auditory centre, by which they are regulated (8). Excessive Glu has been reported to damage SGNs, inducing sensorineural hearing loss and auditory neuropathy (13). Auditory neuropathy is a disease, in which the inner hair cells fail to transmit acoustic information to the brain. This disorder clinically presents with elevated hearing thresholds without a parallel loss of otoacoustic emissions, for which damage to the SGNs may be the cause (3). Increased Glu binds with Glu receptors, including NMDAR2 and mGluRIs, and initiates calcium regulation and oxidative damage, injuring the SGNs (14), which leads to subsequent hearing loss. In the present study, ABR threshold shifts increased with increasing concentrations of perfused Glu. The 20 and $40 \mathrm{mM}$ Glu groups had a greater shift, between $4 \mathrm{kHz}$ and $32 \mathrm{kHz}$, indicating damage mediated by Glu. The number of SGNs was significantly reduced with increasing concentrations of Glu, and TEM identified heterochromatin surrounding the nuclei, which confirmed the damage to the SGNs by excessive Glu and demonstrated a successful model of SGN-damage. These results provide support in further examining the mechanisms of auditory neuropathy. Investigating higher concentrations of Glu may complete experiment data and provide clearer presentation of Glu-damaged SGNs.

Caspase has been a focus of early investigations of cochlea sensory cells. Noise and ototoxic drugs can induce caspase upregulation in outer hair cells, however, Steinbach and Lutz observed that addition of the Z-VAD-FMK caspase inhibitor to SGN cultures prevents the apoptosis of SGNs (14). Abaamrane et al injected Z-VAD-FMK into the cochlea, which resulted in incomplete recovery in deafness, following detonation (15). In the present study, qPCR revealed that the levels of caspase 3 were not upregulated in response to Glu perfusion or SGN culture, which supported the hypothesis that the caspase pathway has a limited effect on Glu toxicity in SGNs (14). However, the RT-qPCR results demonstrated that the mRNA expression levels of AIF increased following Glu treatment, compared with the control group, and the immunostaining assay indicated that AIF was translocated into the nuclei at Glu concentrations of $\geq 20 \mathrm{mM}$ perfusion, in the perfusion and cultured SGNs. These results demonstrated that AIF may be induced in SGN apoptosis and contribute to Glu-mediated SGN injury in sensory hearing loss and auditory neuropathy.

Calpain has been identified as being important in the association between Glu toxicity and AIF in apoptosis (16). Vosler et al demonstrated that calpain can be activated by apoptotic signals, releasing AIF from the inner mitochondrial membrane into the cytoplasm (17), while other studies have observed that AIFis then transferred between the cytoplasm and the nucleus to induce nuclear DNA fragmentation $(3,18)$, which results in an influx of $\mathrm{Ca}^{2+}$ into the cytoplasm through the ion channels of the NMDA Glu receptors and the activation of calpain. In the RT-qPCR results of the present study, the expression of calpain was upregulated subsequent to Glu acting on the SGNs via Glu perfusion or following the addition of Glu to cultured SGNs, highlighting the important role of calpain. TEM demonstrated morphological alterations of the endoplasmic reticulum in the SGNs, and suggested that endogenous $\mathrm{Ca}^{2+}$ was involved in calpain activation in the cytoplasm (19). The results suggested that the calpain-AIF upregulation pathway was important in Glu-mediated damage of SGNs.

In conclusion, the present study demonstrated an explanation for the mechanism underlying Glu-mediated SGN damage in tympanic canal perfusion and in vitro SGNs intervention. This may assist in understanding the relevant mechanisms of auditory neuropathy.

\section{Acknowledgements}

This study was supported by grants from the Major State Basic Research Development Program of China (973 Project; grant no. 2011CB504505) and the National Natural Science Foundation of China (grant nos. 81120108008, 30930098, 81300832 and 81271069).

\section{References}

1. Mizukoshi S, Nakazawa M, Sato K, Ozaki T, Metoki T and Ishiguro S: Activation of mitochondrial calpain and release of apoptosis-inducing factor from mitochondria in RCS rat retinal degeneration. Exp Eye Res 91: 353-361, 2010.

2. Chen Q, Paillard M, Gomez L, et al: Activation of mitochondrial $\mu$-calpain increases AIF cleavage in cardiac mitochondria during ischemia-reperfusion. Biochem Biophys Res Commun 415: 533-538, 2011

3. Zhu C, Wang X, Deinum J, et al: Cyclophilin A participates in the nuclear translocation of apoptosis-inducing factor in neurons after cerebral hypoxia-ischemia. J Exp Med 204: 1741-1748, 2007.

4. Joseph B, Marchetti P, Formstecher P, Kroemer G, Lewensohn R and Zhivotovsky B: Mitochondrial dysfunction is an essential step for killing of non-small cell lung carcinomas resistant to conventional treatment. Oncogene 21: 65-77, 2002.

5. Norberg E, Gogvadze V, Ott M, et al: An increase in intracellular $\mathrm{Ca}_{2}{ }^{+}$is required for the activation of mitochondrial calpain to release AIF during cell death. Cell Death Differ 15: 1857-1864, 2008.

6. Yuan Y, Shi F, Yin Y, et al: Ouabain-induced cochlear nerve degeneration: synaptic loss and plasticity in a mouse model of auditory neuropathy. J Assoc Res Otolaryngol 15: 31-43, 2014.

7. Zhang YM, Ma B, Gao WY, Wen W and Liu HY: Role of glutamate receptors in the spiral ganglion neuron damage induced by acoustic noise. Acta Physiologica Sinica 59: 103-110, 2007 (In Chinese).

8. Starr A: Auditory neuropathy and inner hair cell and synapses. Zhonghua Er Bi Yan Hou Tou Jing Wai Ke Za Zhi 43: 323-326, 2008 (In Chinese).

9. Fu Y, Ding D, Wei L, Jiang H and Salvi R: Ouabain-induced apoptosis in cochlear hair cells and spiral ganglion neurons in vitro. BioMed Res Int 2013: 628064, 2013.

10. Schmutzhard J, Glueckert R, Pritz C, et al: Sepsis otopathy: experimental sepsis leads to significant hearing impairment due to apoptosis and glutamate excitotoxicity in murine cochlea. Dis Model Mech 6: 745-754, 2013. 
11. Rebillard G and Bryant GM: Effects of in vivo perfusion of glutamate dehydrogenase in the guinea pig cochlea on the VIIIth nerve compound action potential. Brain Res 494: 379-382, 1989.

12. Liu W, Fan Z, Han Y, Zhang D, Li J and Wang H: Intranuclear localization of apoptosis-inducing factor and endonuclease $G$ involves in peroxynitrite-induced apoptosis of spiral ganglion neurons. Neurol Res 34: 915-922, 2012.

13. Jiang M, Sun H and Zhang YQ: Excitotoxic effect of glutamate on the afferent neurons in guinea pigs. Zhong Nan Da Xue Xue Bao Yi Xue Ban 29: 170-173, 2004 (In Chinese).

14. Steinbach S and Lutz J: Glutamate induces apoptosis in cultured spiral ganglion explants. Biochem Biophys Res Commun 357: $14-19,2007$.

15. Abaamrane L, Raffin F, Schmerber S and Sendowski I: Intracochlear perfusion of leupeptin and z-VAD-FMK: influence of antiapoptotic agents on gunshot-induced hearing loss. Eur Arch Otorhinolaryngol 268: 987-993, 2011.
16. Ozaki T, Yamashita T and Ishiguro S: Mitochondrial m-calpain plays a role in the release of truncated apoptosis-inducing factor from the mitochondria. Biochim Biophys Acta 1793: 1848-1859, 2009.

17. Vosler PS, Sun D, Wang S, et al: Calcium dysregulation induces apoptosis-inducing factor release: cross-talk between PARP-1- and calpain-signaling pathways. Exp Neurol 218: 213-220, 2009

18. Sanges D, Comitato A, Tammaro R and Marigo V: Apoptosis in retinal degeneration involves cross-talk between apoptosis-inducing factor (AIF) and caspase-12 and is blocked by calpain inhibitors. Proc Natl Acad Sci USA 103: 17366-17371, 2006.

19. Norberg E, Gogvadze V, Ott M, et al: An increase in intracellular $\mathrm{Ca} 2+$ is required for the activation of mitochondrial calpain to release AIF during cell death. Cell Death Differ 15: 1857-1864, 2008. 